\title{
ARF: a versatile DNA damage response ally at the crossroads of development and tumorigenesis
}

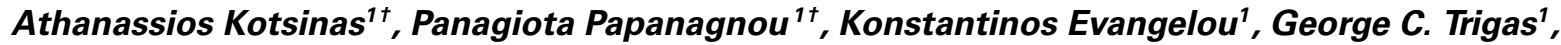 Vassiliki Kostourou" ${ }^{2}$, Paul Townsend ${ }^{3,4}$ and Vassilis G. Gorgoulis ${ }^{1,3,4,5}$ *}

\author{
Molecular Carcinogenesis Group, Department of Histology and Embryology, School of Medicine, University of Athens, Athens, Greece \\ 2 Vascular Adhesion Lab, Biomedical Sciences Research Center Alexander Fleming, Athens, Greece \\ ${ }^{3}$ Faculty Institute of Cancer Sciences, University of Manchester, Manchester Academic Health Science Centre, Manchester, UK \\ ${ }^{4}$ Manchester Centre for Cellular Metabolism, University of Manchester, Manchester Academic Health Science Centre, Manchester, UK \\ ${ }^{5}$ Biomedical Research Foundation, Academy of Athens, Athens, Greece
}

\section{Edited by:}

Antonio Porro, University of Zurich, Switzerland

\section{Reviewed by:}

Triantafillos Liloglou, University of Liverpool, UK

Ken-ichi Yoshioka, National Cancer Center Research Institute, Japan

\section{*Correspondence:}

Vassilis G. Gorgoulis, Molecular

Carcinogenesis Group, Department of Histology and Embryology, School of Medicine, University of Athens, 75

Mikras Asias Street, Athens, Greece e-mail:vgorg@med.uoa.gr

${ }^{\dagger}$ Athanassios Kotsinas and Panagiota Papanagnou have contributed equally to this work.
Alternative reading frame (ARF) is a tumor suppressor protein that senses oncogenic and other stressogenic signals. It can trigger p53-dependent and -independent responses with cell cycle arrest and apoptosis induction being the most prominent ones. Other ARF activities, particularly p53-independent ones, that could help in understanding cancer development and provide potential therapeutic exploitation are underrated. Although ARF is generally not expressed in normal tissues, it is essential for ocular and male germ cells development. The underlying mechanism(s) in these processes, while not clearly defined, point toward a functional link between ARF, DNA damage and angiogenesis. Based on a recent study from our group demonstrating a functional interplay between ataxia-telangiectasia mutated (ATM) and ARF during carcinogenesis, we discuss the role of ARF at the crossroads of cancer and developmental processes.

Keywords: ARF, ATM, angiogenesis, ocular development, vascular network, involution, meiosis, spermatogenesis

\section{INTRODUCTION}

The ARF ( $14^{\mathrm{ARF}}$ in humans, $\mathrm{p} 19^{\mathrm{ARF}}$ in mice) tumor suppressor is encoded by the INK4A/ARF locus that also harbors another onco-suppressor, namely the cyclin-dependent kinase inhibitor p16 ${ }^{\mathrm{INK} 4 \mathrm{~A}}$ (Quelle et al., 1995; Sherr, 2006). The p16 ${ }^{\mathrm{INK} 4 \mathrm{~A}}$ protein maintains $\mathrm{pRB}$ in an active form to inhibit E2F activity (Tsantoulis and Gorgoulis, 2005; Sherr, 2006). In this way S-phase entry and therefore cell division is prevented (Sherr, 2006). On the other hand, ARF is a "sensor" of various stresses including oncogenic ones, like aberrant expression of Myc, E1A and RAS (de Stanchina et al., 1998; Zindy et al., 1998; Palmero et al., 1999). Other stresses that can also activate ARF are oxidative stress and heat shock (Damalas et al., 2011; Liontos et al., 2012). In response it can act both in p53-dependent and -independent manners (Weber et al., 2000; Kotsinas et al., 2014), triggering either growth arrest or apoptosis to counteract abnormal cell proliferation (Sherr, 2006). Apart from cancer (Sherr, 2006), accumulating data highlight ARF as a versatile protein implicated in various physiological processes including developmental ones

Abbreviations: ARF, alternative reading frame; ATM, ataxia-telangiectasia mutated; DDR, DNA damage response; DMSO, dimethyl sulfoxide; DSBs, doubled-strand breaks; FSH, follicle-stimulating hormone; HA, hyaloid artery; HIF1 $\alpha$, hypoxiainducible factor $1 \alpha ; \mathrm{HR}$, homologous recombination; HVS, hyaloid vascular system; IRES, internal ribosome entry sequence; LH, luteinizing hormone; MEFs, mouse embryonic fibroblasts; MVD, microvessel density; NPM/B23, nucleophosmin; NSCLC, non-small cell lung cancer; P, postnatal day; PDGF $\beta$, platelet-derived growth factor $\beta$; PDGFR $\beta$, platelet-derived growth factor receptor $\beta$; PHPV, persistent hyperplastic primary vitreous; $p R b$, retinoblastoma protein; sh, short-hairpin; VEGF, vascular endothelial growth factor; YFP, yellow fluorescent protein.
(Thornton et al., 2005; Gromley et al., 2009; Churchman et al., 2011), immunomodulation (Través et al., 2012) and ribosomal ribonucleic acid (rRNA) maturation (Sugimoto et al., 2003), as well as pathological ones, such as atherogenesis (GonzálezNavarro et al., 2010). Most of the best known ARF functions are p53-dependent ones (Sherr, 2006), while independent activities seem to be underrated.

Deficiency of ARF or p53, has revealed different phenotypes in mice. Specifically, ARF-null animals mainly develop sarcomas, whereas p53-null animals are predominantly characterized by the evolvement of lymphomas (Kamijo et al., 1999). This finding was among the first experimental indications that ARF and p53 can signal independently of each other and not necessarily in a strict linear signaling pathway. Therefore, they may fulfill different tasks in tumor surveillance. Moreover, recent evidence from our group has highlighted the functional significance of a cross-talk among ARF and ATM (Velimezi et al., 2013; Kotsinas et al., 2014) and how ARF can act as an "auxiliary" tumor suppressive mechanism throughout cancer progression in case the DDR pathway is compromised (Velimezi et al., 2013).

In various normal tissues ARF is not expressed. Striking exceptions are the developing oculus (eye), testicular tissue and umbilical arteries (Thornton et al., 2005; Freeman-Anderson et al., 2009; Gromley et al., 2009; Churchman et al., 2011). The underlying mechanism(s) taking place in these tissues, while not clearly defined, point toward a functional link between ARF, DNA damage and angiogenesis. 
Considering the ATM and ARF interplay in carcinogenesis (Velimezi et al., 2013), we discuss in this article the role of ARF at the crossroads of cancer and developmental processes. We present the current knowledge regarding the role of ARF in development, particularly during spermatogenesis and ocular development in mice. Furthermore, we provide data (including unpublished ones) consolidating the notion that the interference with vascular dynamics accounts for a novel, inherent p53-independent tumor suppressive property of ARF that could be therapeutically exploited in p53-deficient tumors.

\section{THE ROLE OF ARF IN MALE GERM CELL DEVELOPMENT: A MATTER OF PRESERVING GENOMIC INTEGRITY}

Spermatogenesis is a spatio-temporally coordinated process by which undifferentiated spermatogonia (i.e., the stem cell population of germinal cells residing on the basement membrane of semiferous tubules) evolve into spermatocytes in the lumen through a series of mitotic and meiotic cellular divisions (Cooke and Saunders, 2002). In mice, Gromley etal. (2009) reported that in this developmental process ARF is selectively expressed in mitotic spermatogonia, but not in intratubular spermatocytes that stain positive for meiotic markers (Figure 1). Intriguingly, in the absence of ARF the testicles of mice exhibit atrophy and produce much lower quantity of sperm compared to wild-type animals. These phenotypic changes are accompanied by increased levels of apoptosis in germ cells during all their developmental stages. Of note, abolishment of this transient expression of ARF during spermatogenesis is sufficient to compromise this developmental process throughout the whole reproductive life of mice (Gromley et al., 2009).

Subsequent experiments carried out by the same research group demonstrated that ARF is essential for normal meiotic progression and survival of spermatocytes via initiation of a feed-forward program in their progenitors, the spermatogonia. Interestingly, ARF expression in spermatogonia did not exert an anti-proliferative effect, as they also expressed cyclin D1. Testicular atrophy and reduced production of mature sperm in ARF-deficient mice was not mechanistically related to a disturbed pituitary-gonadal axis and deregulated levels of circulating FSH or LH. Rather, in the absence of ARF a marked increase in the number of spermatocytes undergoing p53-dependent apoptosis at the stage of pachytene of prophase I was observed (Churchman et al., 2011).

Of note, during HR in meiosis the topoisomerase-II like Spo11 enzyme normally causes DSBs, which in turn trigger the activation of ATM and the generation of $\gamma-\mathrm{H} 2 \mathrm{AX}$ foci selectively at the leptotene and zygotene stages (Inagaki et al., 2010). At the pachytene stage when synapsis of homologous chromosomes has been completed, $\gamma$-H2AX foci are normally not detected. However, in situ analyses showed that in an ARF deficient background, the number of $\gamma-\mathrm{H} 2 \mathrm{AX}$ foci in pachytene spermatocytes is significantly increased (Churchman et al., 2011). The latter observation was therefore suggestive of meiotic defects that have deleterious effects on spermatocytic genomic integrity. Additional evidence for meiotic abnormalities included the identification of asynaptic regions as well as decreased number of foci of the Rad51 and Dmcl recombinases known to be associated with the repair of DSBs that occur during HR. Overall, the authors concluded that ARF through a yet poorly understood mechanism, interferes with HR to preserve the fidelity of meiosis in spermatocytes and to protect them from DNA damage and p53-dependent apoptosis (Churchman et al., 2011). Whether the apoptotic death of spermatocytes is a consequence of DNA damage per se or not, remains to be elucidated. Inhibition rather than induction of p53-dependent apoptosis by ARF is a unique feature of male germ cells and reveals an opposite to the well-established p53-mediated pro-apoptotic role of ARF in somatic cells (Lowe and Sherr, 2003).

Importantly, ARF is not the only DNA damage-related protein that interferes with the spermatogonial program (Figure 1). The DDR kinase ATM is actually essential for the maintenance of undifferentiated spermatogonia and for retaining their stemness (Barlow et al., 1998; Takubo et al., 2008). In murine testicles, ATM deficiency progressively results in the depletion of undifferentiated spermatogonia. This is functionally associated with cell cycle arrest, loss of genomic integrity and defects at the pre-meiotic level (Barlow et al., 1996, 1998; Elson et al., 1996; Xu et al., 1996; Takubo et al., 2008). Mechanistically, the absence of ATM is linked to the accumulation of DNA damage and the activation of an ARF/p53/p21 ${ }^{\text {AF1/Cip } 1 ~-~ d e p e n d e n t ~ g r o w t h ~ r e s t r i c t i v e ~ p a t h w a y . ~}$ Notably, in transplantation assays where spermatogonia are delivered into the seminiferous tubules of mutant mouse strains that exhibit defective spermatogenesis, $\mathrm{p} 21^{\mathrm{WAF} 1 / \mathrm{Cip} 1}$ deficiency is able to restore spermatogonial repopulation ability in an ATM-null background (Takubo et al., 2008).

Taken together, all the above data pinpoint that both ARF and ATM are critical factors for the maintenance of spermatogonia and survival of their progeny during male germ cell development (Figure 1; Barlow et al., 1996; Takubo et al., 2008; Churchman et al., 2011). This common feature parallels with their same function as tumor suppressors in somatic cells. Nevertheless, the imposed outcomes are different in each cell type, suggesting a functional bimodality. In somatic cells ATM and ARF induce cell growth restrictive or apoptotic routes, whereas in spermatogonia they do not interfere with their ability to proliferate. It is rather their depletion that leads to such cellular responses. A further issue, stemmed from the ability of ATM to regulate ARF turnover (Velimezi et al., 2013), is whether this functional link may operate in male germ cells. As demonstrated, in response to irradiation ARF protein in spermatogonia is markedly downregulated (Velimezi et al., 2013). Evidence was also provided by Takubo etal. (2008) showing that in ATM null spermatogonia there is a higher activation of ARF, supporting the interconnection between ATM and ARF. Nevertheless, details on how this link endorses this developmental process require further clarifications.

\section{ARF AS A REGULATOR OF THE VASCULAR NETWORK IN DEVELOPMENT AND TUMORIGENESIS}

Apart from the male germ cell development in mice as presented above, ARF also plays a central role in the murine ocular development. In mice models it was shown that ARF is required for the maturation of the primary vitreous into the secondary vitreous; an avascular jelly like substance within the developing oculus. The expression of ARF in the vitreous is postnatally induced up to P5, in order to trigger the involution of HVS, a transient anatomical 

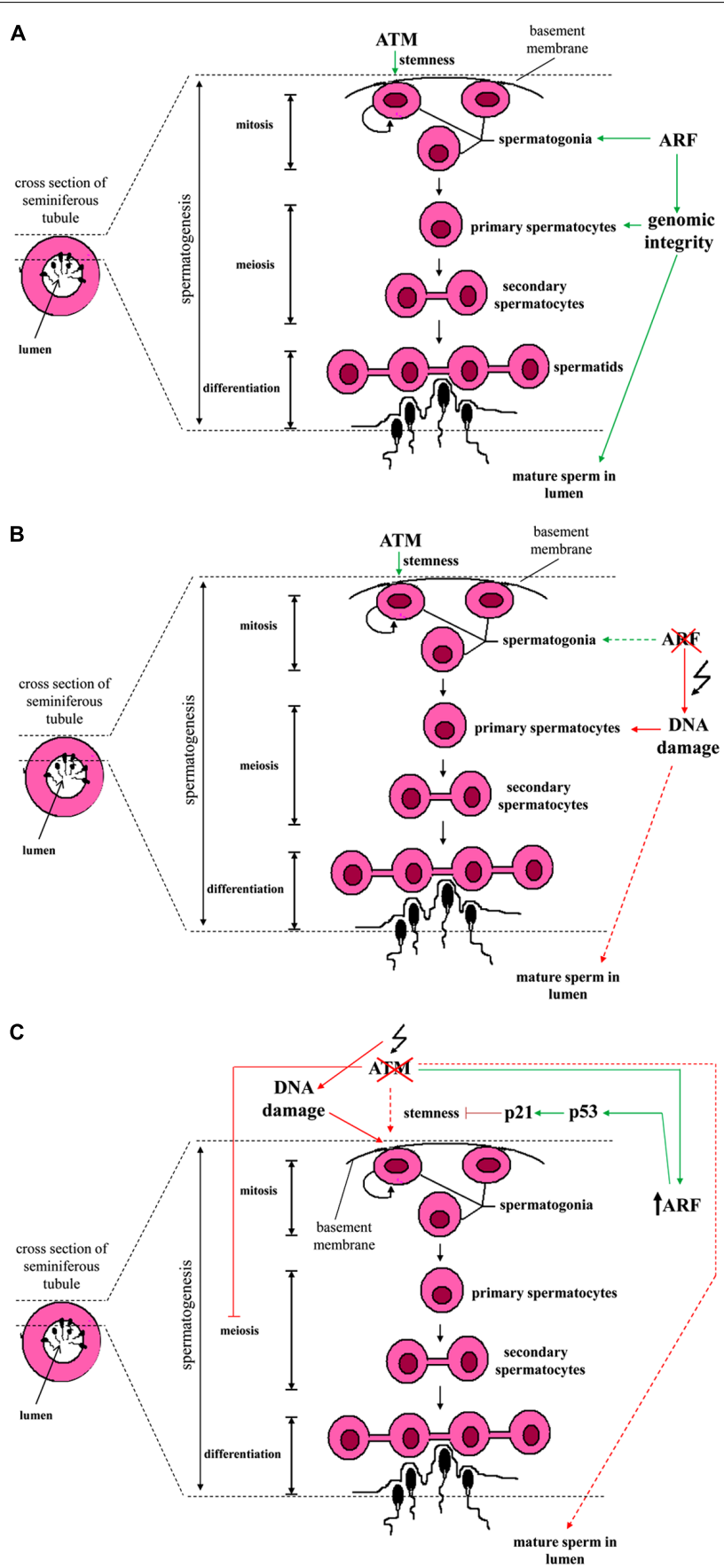

FIGURE 1 | Schematic presentation of the spermatogenesis stages across the wall of a seminiferous tubule and the interrelations with ATM and ARF. (A) Spermatogonia are found in close proximity to the basement membrane of a seminiferous tubule. A subpopulation of spermatogonia exhibits stem cell ability and self-renews via mitotic divisions. ATM kinase is essential for their stemness (symbolized by a semicircular shape). Some of the spermatogonia eventually differentiate into primary spermatocytes. The latter, undergo meiosis to give rise to secondary spermatocytes which in turn, form spermatids (connected via cytoplasmic bridges). Spermatids engage a series of cytodifferentiative programs and sperm is finally formed. ARF expression in spermatogonia is required in order to prevent the occurrence of DNA damage in meiotic primary spermatocytes (at the stage of pachytene) through participating in a feed-forward program. (B) In the absence of ARF, genomic integrity in primary spermatocytes is threatened and the production of mature sperm released in the lumen is compromised. (C) Upon ATM deficiency, spermatogonia loose their genomic integrity and ARF undergoes upregulation. Consequently, an ARF-mediated p53/p21 WAF1/Cip1 growth restrictive pathway counteracts spermatogonial stemness. Due to the fact that ATM deficiency hampers normal spermatocytic meiotic progression (at prophase I), sperm production is compromised (Barlow etal., 1996, 1998; Xu etal., 1996). The different subtypes of spermatogonial cells, Sertoli cells that support spermatogonia, stages and phases of meiosis, the different stages of spermatid differentiation as well as ploidy of cells are not shown here for reasons of simplicity (Colored lines depict ATM and/or ARF effects on spermatogenesis. Dashed colored lines denote weak effect or weak activation. Red lines represent adverse effect, while green ones correspond to physiologic functions). 
entity in the developing oculus that has to regress during P6-P10 (McKeller et al., 2002). ARF promotes HVS involution through restricting the accumulation of mural cells that cover the vessels and contributes to the preservation of their stability in a PDGFR $\beta$ dependent/p53-independent manner (Silva et al., 2005; Gromley et al., 2009). Overall, two models have been proposed to explain the mechanistic basis by which ARF controls the vascular network dynamics. According to the first model, when ARF is induced by unknown yet upstream developmental signals it suppresses PDGFR $\beta$ expression via uncharacterized mediators. In this way, ARF restricts mural cell proliferation. In the second scenario, ARF acts as a cell fate determinant during the maturation of mural cells to shut off PDGFR $\beta$ expression and force them to differentiate into a type of perivascular cells that selectively support transient vessels (Thornton et al., 2005).

Notably, phenotypic characteristics exhibited by ARF deficient mice are also found in the developmental human ocular disease termed PHPV and include microphthalmia and degenerative alterations in lens (cataractogenesis; McKeller et al., 2002). Overall, these data pinpoint to the existence of an ARF-mediated tightly regulated spatio-temporal angiogenic developmental process. It appears that ARF's role in vascular evolvement is not solely restricted to developmental processes. Rather, data corroborate the notion that ARF exhibits a wider activity in the control of vascular dynamics, which are functionally linked to tumor progression.

"Angiogenic switch" is a prominent feature of tumor progression (Bergers and Benjamin, 2003). In line with the notion that ARF plays a wide role in the modulation of pathways affecting vasculature, an inverse correlation among MVD and ARF in human clinical colon cancer samples has been reported (Kawagishi et al., 2010). Furthermore, ARF/p53 deficient MEFs challenged with oncogenic RAS ${ }^{\mathrm{V} 12}$ when injected as xenografts produce tumors that grow faster relative to similarly treated cells, but retrovirally infected with ARF (Kawagishi et al., 2010). Tumor sections showed in the latter case a lower immunostaining for CD31, a neovascular marker. Exploring mechanistically their findings Kawagishi et al. (2010) determined that ARF suppresses the expression of VEGFA in a p53-independent manner in mouse cell lines. This involves the inhibition of VEGFA translation via the IRES of VEGFA.

Based on our recent finding that ATM controls ARF turnover (Velimezi et al., 2013), we sought to expand these observations in human tumors with inactive p53 and explore for potential therapeutic utilization. Treating the NSCLC cell line H1299 and cervical carcinoma HeLa cells, which do not express functional p53, with the ATM kinase inhibitor Ku55933 in order to stabilize ARF (Velimezi et al., 2013) we observed an inverse relationship among the expression of ARF and VEGF protein (Figure S1A). VEGF plays a central role in tumor angiogenesis (Crinò and Metro, 2014), while ARF can signal in a p53-independent fashion and hence, serve as a "back-up" barrier to tumorigenesis in case that p53 is inactivated (Velimezi et al., 2013). Therefore, upregulating ARF via inhibition of ATM activity may be exploited in p53-deficient human tumors as a novel anti-angiogenic therapeutic approach. In a next step, we investigated whether ARF opposes tumor angiogenesis in vivo. To address this issue H1299 cells were xenografted in immunocompromised mice. Sections from the generated tumors were stained with an antibody specific for the endothelial marker CD31, to evaluate MVD. As shown in Figure S1B, MVD is markedly decreased (>twofold) in tumors injected with a lentiviral vector expressing shRNA targeting ATM (ctl-shRNA/Lenti-shATM); a manipulation which upregulates ARF (Velimezi et al., 2013). The observed reduction in MVD was actually an ARF-dependent phenomenon, because MVD in H1299-shARF xenografts upon knocking down of ATM (shARF/Lenti-shATM) was found to be comparable to that estimated in ARF-expressing ones without lentivirusmediated silencing of ATM kinase (ctl-shRNA/Lenti-ctl). Therefore, stabilization of ARF in the absence of ATM activity exhibits anti-angiogenetic effects in vivo, corroborating the claim that this p53-independent ATM/ARF axis could be therapeutically harnessed.

In our experiments we did not examine whether the observed ARF-mediated down-regulation of VEGF levels is associated with an inhibition of IRES-(in)dependent translation of VEGF transcript (Kawagishi et al., 2010), or if it is due to a translationindependent mechanism. For instance, HIFl $\alpha$ is a well-known transcriptional activator of VEGF and human ARF has been demonstrated to mediate the nucleolar sequestration of HIF $1 \alpha$, thereby hindering its ability to drive the expression of its target genes (Fatyol and Szalay, 2001). It would be a challenging future task to uncover the whole mechanistic spectrum that underlies the observed mutual exclusive expression among ARF and VEGF.

In a murine model of multi-stage pancreatic neuroendrocrine tumorigenesis where the SV-40 T-antigen is expressed in $\beta$ cells, ARF deficiency was found to significantly accelerate tumor progression through promoting the angiogenic switch (Ulanet and Hanahan, 2010). In the absence of ARF the tumor burden was increased fivefold along with a higher number of angiogenic lesions. From a mechanistic perspective, in this malignancy ARF seems to act via engaging mainly p53-independent routes, while VEGF was not involved (Ulanet and Hanahan, 2010). A more recent study showed that ARF blocks the development of angiosarcomas associated with the exposure to the carcinogen urothene, possibly in a p53-dependent fashion (Busch et al., 2012). Intriguingly, it was hypothesized that ARF affects the proliferation of endothelial cells in adult mice. Hence, the inhibition of tumor angiogenesis and vascular malignancy possibly represent two discrete aspects of ARF's tumor-suppressive activity.

\section{CONCLUSIONS AND PROSPECTS}

Collectively, we highlight underrated functions of ARF, positioned at the crossroads of tumor suppression (Weber et al., 2000; Tago et al., 2005; Kawagishi et al., 2010) and development (McKeller etal., 2002; Churchman etal., 2011; Figure 2), that could be exploited at the therapeutic level, especially in tumors with non-functional p53.

ARF as an onco-suppressor impedes carcinogenesis not only through interfering with cell proliferation and induction of apoptosis, but also via affecting other cancerpromoting processes such as angiogenesis (Kawagishi et al., 2010; Ulanet and Hanahan, 2010). In this context, in tumors with nonfunctional p53 the potential to upregulate ARF in an ATM manner 


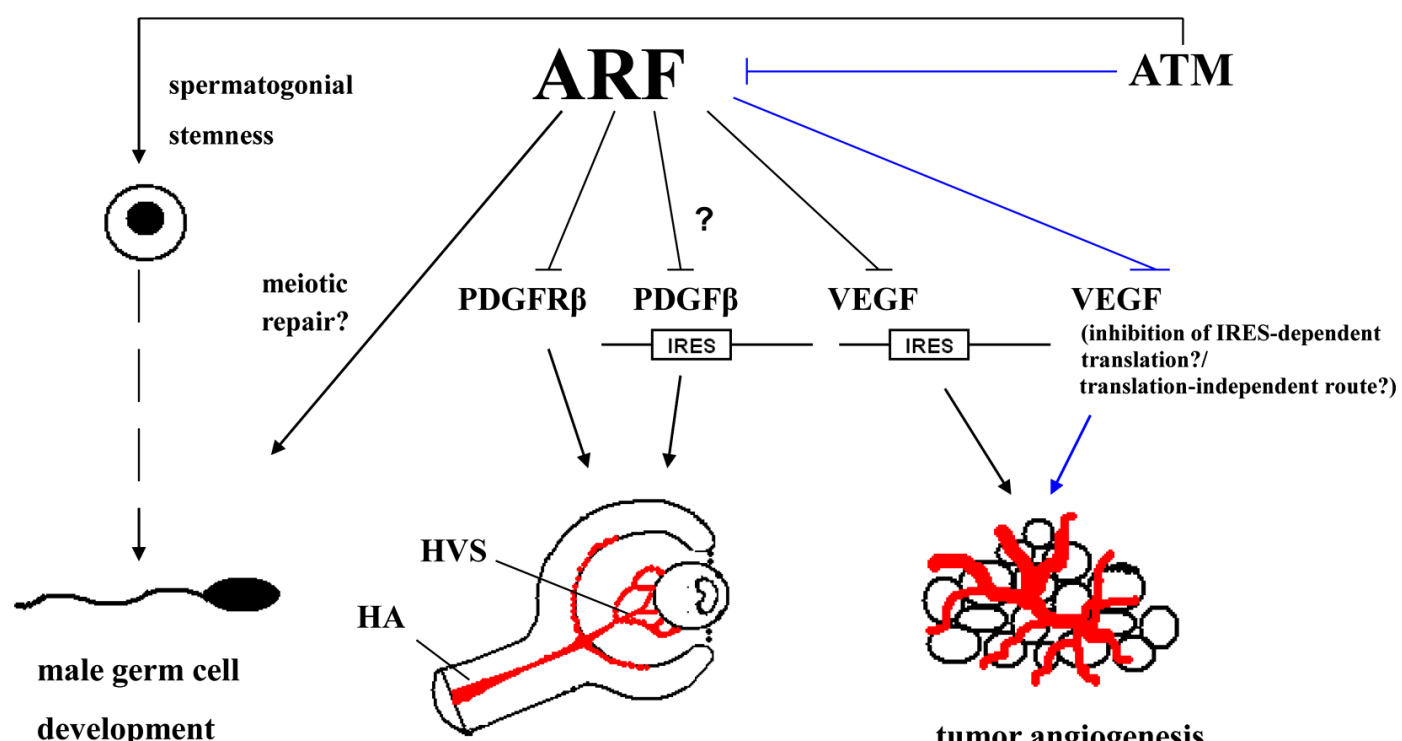

eye development

FIGURE 2 | ARF impinges both on developmental processes (male germ cell development and ocular development in mice) and tumor angiogenesis. ARF is essential for normal spermatogenesis in mice possibly through interacting with the meiotic repair machinery. In the ocular development of the mouse, ARF is required for the involution of the hyaloid vascular system (HVS); a transient network of vessels that provides nutrients to the developing oculus. This is accomplished via a pathway in which ARF blocks platelet-derived growth factor receptor $\beta$ (PDGFR $\beta$ )-dependent signaling which in turn, is necessary for the investment of vessels by mural cells and their maintenance. This may also be possibly regulated via the ARF-dependent inhibition of internal ribosome entry site (IRES)-mediated translation of PDGFR $\beta$. ARF can also suppress the
IRES-mediated translation of vascular endothelial growth factor A (VEGFA), thereby inhibiting tumor angiogenesis. Unpublished data indicate that the stabilization of endogenous ARF upon inhibition of ataxia-telangiectasia mutated (ATM) kinase activity can also result in a decrease in VEGF levels; although it is not known whether the underlying mechanism involves the suppression of IRES-mediated translation of VEGF or an IRES-independent route. It may even involve a route that is not associated with the control of VEGF at the translational level. (The ATM/ARFNEGF pathway is shown by lines.) ATM kinase itself, independently of ARF, has also been incriminated in pathological angiogenesis in adult mouse oculus and cancer (Kerr and Byzova, 2012), but these effects are not depicted here for reasons of clarity. (HA, hyaloid artery.)
(Velimezi et al., 2013), could be utilized as an anti-angiogenic "tool" in cancer management (Figure $\mathbf{S 1}$ ). This prospective therapeutic modality may be enhanced if combined with anti-VEGF or other anti-angiogenic factors, like tyrosine kinase inhibitors, that are currently used (Ferrara et al., 2004; Randall and Monk, 2010; Eisen et al., 2012). On one hand, such a dual treatment might lead to a synergistic outcome, possibly lethality, and on the other hand, it may allow the reduction of the administration doses of such compounds to avoid side-effects (Randall and Monk, 2010; Eisen et al., 2012). It should be noted that although the ATM inhibitor Ku55933 is highly selective toward ATM, its bioavailiability is low due to its pharmacokinetic properties (Golding et al., 2009). However, new ATM inhibitors, such as KU-60019, exhibiting a higher pharmacological profile have been released (Golding et al., 2009), rendering the proposed therapeutic approach feasible.

Another option in order to therapeutically exploit the aforementioned ARF/VEGF pathway would be the usage of synthetic ARF peptides comprising ARF's amino-terminal residues 2-14 that mediate all the biological effects of ARF, including the antigrowth ones (Saporita et al., 2007). The therapeutic exploitation of the p53-independent ARF/VEGF axis is of major clinical importance since p53 is inactivated in $\sim 50 \%$ of human cancers (Sherr, 2006).
A further aspect that needs to be addressed is the role of ARF in spermatogenesis. Deciphering the poorly defined ability of ARF to cross-talk with components of the HR mechanism during this process could provide new insights on other underrated functions of ARF. Specifically, in the case of male germ cells ARF contributes to their genomic integrity during their maturation, but the exact mechanism(s) is still lacking (Churchman et al., 2011). Even more, as ATM also participates in this process and since they are functionally linked (Velimezi et al., 2013), the exact way their function is coordinated throughout this process remains to be defined.

Finally, taking into consideration the role of ARF not only in the involution of HVS in the developing oculus (Thornton et al., 2005) but also in the involution of the mammary gland (Yi et al., 2004), it is plausible that ARF plays an even wider role than that of a tumor suppressor by acting as a potent "tissue remodeling factor" controlling transient histological structures. Studies toward this direction are essential and could open a new research field related with ARF.

\section{ACKNOWLEDGMENTS}

This project has received funding from the European Union's Seventh Framework Program for research, technological development and demonstration under grant agreement No 284460. 
Dr Athanassios Kotsinas is a recipient of an Empeirikeion Foundation fellowship.

\section{SUPPLEMENTARY MATERIAL}

The Supplementary Material for this article can be found online at: http://www.frontiersin.org/journal/10.3389/fgene.2014.00236/ abstract

Figure S1 | ARF demonstrates a p53-independent anti-angiogenic activity in a malignant environment. (A) Evidence for a p53-independent ATM/ARFNEGF pathway. Human ARF protein (mouse monoclonal antibody, 1:100 dilution, DCS-240, \#ab49166, Abcam) was up-regulated in the presence of the selective ATM inhibitor Ku55933 (Ku, \#118500, Calbiochem, MERCK) and as previously shown biologically effective Nelimezi et al., 2013). This was associated with a decrease in VEGF protein (rabbit polyclonal antibody, 1:100 dilution, A-20, \#sc-152, SantaCruz) levels as assessed by immunoblot analysis in human cancer cells H1299 and HeLa, both of which are devoid of functional p53. Actin (rabbit polyclonal antibody, 1:1000 dilution, Cell Signaling Technology Inc., \#4967) served as a loading control. (ATMi: treatment with the Ku55933 chemical inhibition of ATM activity). (B) ARF-dependent decreased microvessel density in a malignant environment. The non-small cell lung cancer (NSCLC) cells $\mathrm{H} 1299$, which do not express a functional p53 were xenografted in immunocompromised mice and formed tumors (Nelimezi et al., 2013). Immunohistochemical (IHC) analysis was carried out using an anti-CD31 antibody in order to evaluate mean microvessel density (MVD) in sections from tumor tissue. MVD is more than twofold decreased in tumors that were injected with a lentiviral vector expressing short-hairpin (sh) RNA targeting ATM (ctl-shRNA/Lenti-shATM) where ARF is known to be upregulated. On the contrary, the MVD in H1299-shARF xenografts, where ATM has been silenced (shARF/Lenti-shATM), is not decreased. Rather, it is comparable to those estimated in ARF-expressing xenografts without lentivirus-mediated silencing of ATM kinase (ctl-shRNA/Lenti-ctl).

\section{REFERENCES}

Barlow, C., Hirotsune, S., Paylor, R., Liyanage, M., Eckhaus, M., Collins, F., et al. (1996). Atm-deficient mice: a paradigm of ataxia telangiectasia. Cell 86, 159-171. doi: 10.1016/S0092-8674(00)80086-0

Barlow, C., Liyanage, M., Moens, P. B., Tarsounas, M., Nagashima, K., Brown, K., et al. (1998). Atm deficiency results in severe meiotic disruption as early as leptonema of prophase I. Development 125, 4007-4017.

Bergers, G., and Benjamin, L. E. (2003). Tumorigenesis and the angiogenic switch. Nat. Rev. Cancer 3, 401-410. doi: 10.1038/nrc1093

Busch, S. E., Gurley, K. E., Moser, R. D., and Kemp, C. J. (2012). ARF suppresses hepatic vascular neoplasia in a carcinogen-exposed murine model. J. Pathol. 227, 298-305. doi: 10.1002/path.4024

Cooke, H. J., and Saunders, P. T. (2002). Mouse models of male infertility. Nat. Rev. Genet. 3, 790-801. doi: 10.1038/nrg911

Churchman, M. L., Roig, I., Jasin, M., Keeney, S., and Sherr, C. J. (2011). Expression of arf tumor suppressor in spermatogonia facilitates meiotic progression in male germ cells. PLoS Genet. 7:e1002157. doi: 10.1371/journal.pgen.10 02157

Crinò, L., and Metro, G. (2014). Therapeutic options targeting angiogenesis in nonsmall cell lung cancer. Eur. Respir. Rev. 23, 79-91. doi: 10.1183/09059180.000 08913

Damalas, A., Velimezi, G., Kalaitzakis, A., Liontos, M., Papavassiliou, A. G., Gorgoulis, V., et al. (2011). Loss of p14 $4^{\mathrm{ARF}}$ confers resistance to heat shock- and oxidative stress-mediated cell death by upregulating $\beta$-catenin. Int. J. Cancer 128 , 1989-1995. doi: 10.1002/ijc.25510

de Stanchina, E., McCurrach, M. E., Zindy, F., Shieh, S. Y., Ferbeyre, G., Samuelson, A. V., et al. (1998). E1A signaling to p53 involves the p19 $9^{\text {ARF }}$ tumor suppressor. Genes Dev. 12, 2434-2442. doi: 10.1101/gad.12.15.2434

Eisen, T., Sternberg, C. N., Robert, C., Mulders, P., Pyle, L., Zbinden, S., et al. (2012). Targeted therapies for renal cell carcinoma: review of adverse event management strategies. J. Natl. Cancer Inst. 104, 93-113. doi: 10.1093/jnci/ djr511

Elson, A., Wang, Y., Daugherty, C. J., Morton, C. C., Zhou, F., Campos-Torres, J., et al. (1996). Pleiotropic defects in ataxia-telangiectasia protein-deficient mice. Proc. Natl. Acad. Sci. U.S.A. 93, 13084-13089. doi: 10.1073/pnas.93.23. 13084

Fatyol, K., and Szalay, A. A. (2001). The p14ARF tumor suppressor protein facilitates nucleolar sequestration of hypoxia-inducible factor-1alpha (HIF-1alpha) and inhibits HIF-1-mediated transcription. J. Biol. Chem. 276, 28421-28429. doi: 10.1074/jbc.M102847200

Ferrara, N., Hillan, K. J., Gerber, H. P., and Novotny, W. (2004). Discovery and development of bevacizumab, an anti-VEGF antibody for treating cancer. Nat. Rev. Drug Discov. 3, 391-400. doi: 10.1038/nrd1381

Freeman-Anderson, N. E., Zheng, Y., McCalla-Martin, A. C., Treanor, L. M., Zhao, Y. D., Garfin, P. M., et al. (2009). Expression of the Arf tumor suppressor gene is controlled by Tgfbeta2 during development. Development 136, 2081-2089. doi: 10.1242/dev.033548

Golding, S. E., Rosenberg, E., Valerie, N., Hussaini, I., Frigerio, M., Cockcroft, X. F., et al. (2009). Improved ATM kinase inhibitor KU-60019 radiosensitizes glioma cells, compromises insulin, AKT and ERK prosurvival signaling, and inhibits migration and invasion. Mol. Cancer Ther. 8, 2894-902. doi: 10.1158/1535-7163.MCT-09-0519

González-Navarro, H., Abu Nabah, Y. N., Vinué, A., Andrés-Manzano, M. J., Collado, M., Serrano, M., et al. (2010). p19ARF deficiency reduces macrophage and vascular smooth muscle cell apoptosis and aggravates atherosclerosis. J. Am. Coll. Cardiol. 55, 2258-2268. doi: 10.1016/j.jacc.2010.01.026

Gromley, A., Churchman, M. L., Zindy, F., and Sherr, C. J. (2009). Transient expression of the Arf tumor suppressor during male germ cell and eye development in Arf-Cre reporter mice. Proc. Natl. Acad. Sci. U.S.A. 106, 6285-6290. doi: 10.1073/pnas.0902310106

Inagaki, A., Schoenmakers, S., and Baarends, W. M. (2010). DNA double strand break repair, chromosome synapsis and transcriptional silencing in meiosis. Epigenetics 5, 255-266. doi: 10.4161/epi.5.4.11518

Kamijo, T., Bodner, S., van de Kamp, E., Randle, D. H., and Sherr, C. J. (1999) Tumor spectrum in ARF-deficient mice. Cancer Res. 59, 2217-2222.

Kawagishi, H., Nakamura, H., Maruyama, M., Mizutani, S., Sugimoto, K., Takagi, M., et al. (2010). ARF suppresses tumor angiogenesis through translational control of VEGFA mRNA. Cancer Res. 70, 4749-4758. doi: 10.1158/00085472.CAN-10-0368

Kerr, B. A., and Byzova, T. V. (2012). The dark side of the oxidative force in angiogenesis. Nat. Med. 18, 1184-1185. doi: 10.1038/nm.2881

Kotsinas, A., Papanagnou, P., Galanos, P., Schramek, D., Townsend, P., Penninger, J. M., et al. (2014). MKK7 and ARF: new players in the DNA damage response scenery. Cell Cycle 13, 1227-1236. doi: 10.4161/cc.28654

Liontos, M., Pateras, I. S., Evangelou, K., and Gorgoulis, V. G. (2012). The tumor suppressor gene ARF as a sensor of oxidative stress. Curr. Mol. Med. 12, 704-715. doi: $10.2174 / 156652412800792633$

Lowe, S. W., and Sherr, C. J. (2003). Tumor suppression by Ink4a-Arf: progress and puzzles. Curr. Opin. Genet. Dev. 13, 77-83. doi: 10.1016/S0959-437X(02) 00013-8

McKeller, R. N., Fowler, J. L., Cunningham, J. J., Warner, N., Smeyne, R. J., Zindy, F., et al. (2002). The Arf tumor suppressor gene promotes hyaloid vascular regression during mouse eye development. Proc. Natl. Acad. Sci. U.S.A. 99, 3848-3853. doi: 10.1073/pnas.052484199

Palmero, I., Pantoja, C., and Serrano, M. (1999). p19ARF links the tumour suppressor p53 to Ras. Nature 395, 125-126. doi: 10.1038/25870

Quelle, D. E., Zindy, F., Ashmun, R. A., and Sherr, C. J. (1995). Alternative reading frames of the INK4a tumor suppressor gene encode two unrelated proteins capable of inducing cell cycle arrest. Cell 83, 993-100. doi: 10.1016/0092-8674(95)90214-7

Randall, L. M., and Monk, B. J. (2010). Bevacizumab toxicities and their management in ovarian cancer. Gynecol. Oncol. 117, 497-504. doi: 10.1016/j.ygyno.2010.02.021

Saporita, A. J., Maggi, L. B. Jr., Apicelli, A. J., and Weber, J. D. (2007). Therapeutic targets in the ARF tumor suppressor pathway. Curr. Med. Chem. 14, 1815-1827. doi: 10.2174/092986707781058869

Sherr, C. J. (2006). Divorcing ARF and p53: an unsettled case. Nat. Rev. Cancer 6, 663-73. doi: 10.1038/nrc1954

Silva, R. L., Thornton, J. D., Martin, A. C., Rehg, J. E., Bertwistle, D., Zindy, F., et al. (2005). Arf-dependent regulation of Pdgf signaling in perivascular cells in the developing mouse eye. EMBO J. 24, 2803-2814. doi: 10.1038/sj.emboj. 7600751 
Sugimoto, M., Kuo, M. L., Roussel, M. F., and Sherr, C. J. (2003). Nucleolar Arf tumor suppressor inhibits ribosomal RNA processing. Mol. Cell. 11, 415-424. doi: 10.1016/S1097-2765(03)00057-1

Tago, K., Chiocca, S., and Sherr, C. J. (2005). Sumoylation induced by the Arf tumor suppressor: a p53-independent function. Proc. Natl. Acad. Sci. U.S.A. 102, 7689-7694. doi: 10.1073/pnas.0502978102

Takubo, K., Ohmura, M., Azuma, M., Nagamatsu, G., Yamada, W., Arai, F., et al. (2008). Stem cell defects in ATM-deficient undifferentiated spermatogonia through DNA damage-induced cell-cycle arrest. Cell Stem Cell 2, 170-182. doi: 10.1016/j.stem.2007.10.023

Thornton, J. D., Silva, R. L., Martin, A. C., and Skapek, S. X. (2005). The Arf tumor suppressor regulates platelet-derived growth factor receptor beta signaling: a new view through the eyes of $A r f^{-/-}$mice. Cell Cycle 4, 1316-1319. doi: $10.4161 /$ cc. 4.10 .2109

Través, P. G., Luque, A., and Hortelano, S. (2012). Macrophages, inflammation, and tumor suppressors: ARF, a new player in the game. Mediators Inflamm. 2012, 568783. doi: $10.1155 / 2012 / 568783$

Tsantoulis, P. K., and Gorgoulis, V. G. (2005). Involvement of E2F transcription factor family in cancer. Eur. J. Cancer 41, 2403-2414. doi: 10.1016/j.ejca.2005.08.005

Ulanet, D. B., and Hanahan, D. (2010). Loss of p19 Arf facilitates the angiogenic switch and tumor initiation in a multi-stage cancer model via p53-dependent and independent mechanisms. PLoS ONE 5:e12454. doi: 10.1371/journal.pone. 0012454

Velimezi, G., Liontos, M., Vougas, K., Roumeliotis, T., Bartkova, J., Sideridou, M., et al. (2013). Functional interplay between the DNA-damage-response kinase ATM and ARF tumour suppressor protein in human cancer. Nat. Cell Biol. 15, 967-977. doi: 10.1038/ncb2795

Weber, J. D., Jeffers, J. R., Rehg, J. E., Randle, D. H., Lozano, G., Roussel, M. F., et al. (2000). p53-independent functions of the p19 ${ }^{A R F}$ tumor suppressor. Genes Dev. 14, 2358-2365. doi: 10.1101/gad.827300
Xu, Y., Ashley, T., Brainerd, E. E., Bronson, R. T., Meyn, M. S., and Baltimore, D. (1996). Targeted disruption of ATM leads to growth retardation, chromosomal fragmentation during meiosis, immune defects, and thymic lymphoma. Genes Dev. 10, 2411-2422. doi: 10.1101/gad.10.19.2411

Yi, Y., Shepard, A., Kittrell, F., Mulac-Jericevic, B., Medina, D., and Said, T. K. (2004). p19ARF determines the balance between normal cell proliferation rate and apoptosis during mammary gland development. Mol. Biol. Cell 15, 23022311. doi: 10.1091/mbc.E03-11-0785

Zindy, F., Eischen, C. M., Randle, D. H., Kamijo, T., Cleveland, J. L., Sherr, C. J., et al. (1998). Myc signaling via the ARF tumor suppressor regulates p53dependent apoptosis and immortalization. Genes Dev. 12, 2424-2433. doi: $10.1101 /$ gad.12.15.2424

Conflict of Interest Statement: The authors declare that the research was conducted in the absence of any commercial or financial relationships that could be construed as a potential conflict of interest.

Received: 30 May 2014; accepted: 03 July 2014; published online: 22 July 2014.

Citation: Kotsinas A, Papanagnou P, Evangelou K, Trigas GC, Kostourou V, Townsend P and Gorgoulis VG (2014) ARF: a versatile DNA damage response ally at the crossroads of development and tumorigenesis. Front. Genet. 5:236. doi: 10.3389/fgene.2014.00236 This article was submitted to Cancer Genetics, a section of the journal Frontiers in Genetics.

Copyright (ㄷ 2014 Kotsinas, Papanagnou, Evangelou, Trigas, Kostourou, Townsend and Gorgoulis. This is an open-access article distributed under the terms of the Creative Commons Attribution License (CC BY). The use, distribution or reproduction in other forums is permitted, provided the original author(s) or licensor are credited and that the original publication in this journal is cited, in accordance with accepted academic practice. No use, distribution or reproduction is permitted which does not comply with these terms. 